\title{
A Review of Sentiment Analysis Techniques
}

\author{
Suzan Hamed \\ Faculty of Graduate Studies for \\ Statistical Research
}

\author{
Mostafa Ezzat, PhD \\ Associate Professor \\ Faculty of Graduate Studies for \\ Statistical Research
}

\author{
Hesham Hefny \\ Vice Deen \\ Faculty of Graduate Studies for \\ Statistical Research
}

\begin{abstract}
The world wide web makes enormous amount of data which forms in users' opinions and emotions about different political and social events etc. sentiment of users which are expressed on the web has a great effect on the readers and politicians. that's because organizations always need to be aware about public opinions for their products and service. social media became a platform to exchange point of views with a reference to sentiment analysis as a text organization which is used to classify expressing emotions in different ways like negative, positive, favorable, and unfavorable. The challenge which faces sentiment analysis is the lack of labeled data in NLP. This review paper describes the latest studies which concern with fulfillment deep learning models to sentiment analysis as deep neural networks, convolutional neural networks, and others to solve various problems.
\end{abstract}

\section{Keywords}

Sentiment Analysis, Deep Learning.

\section{INTRODUCTION}

sentiment analysis or opinion exploration aims to determine the position of the speaker or writer and to know his views on a specific topic. The sentiment analysis contains many different applications in different fields. In the business sector, companies are allowed to collect customer opinions automatically about their products or services. In politics, it can help infer the general trend and reactions to political events, which helps in decision-making. Morale analysis can also be done at many levels, document level, sentence level and subject level.

In the following sections we will covers the detailed about sentiment analysis and deep learning, in section two presents sentiment analysis techniques then in section three we illustrate sentiment analysis model, and then presents the discussion and finally presents the conclusion whereas.

\subsection{Sentiment Analysis}

Morale analysis is one of the most active areas in natural language processing (NLP). It is also a mathematical study of people's feelings and opinions. We always look for the opinions of others whenever we need to decide. This applies not only to individuals, but also to societies and organizations. This allows us to describe feelings, thoughts, emotions and opinions. Our perceptions and imaginations of reality and the choices we make tend to know how others see and value the world through them. Sentiment analysis has been extensively studied in data mining and other areas. Also, it may not be necessary to conduct polls to collect opinions from the general public, as there is a lot of this information available to everyone. In recent years, publications on social media have been able to help companies reshape them, influence public sentiments, and thus have greatly affected our social, political, and other systems. It has become important to collect, study and evaluate opinions. However, finding and evaluating opinions on social media is still a big task because of the proliferation and plurality of sites on social media, as each site contains a large number of opinion text that is difficult to decipher easily in long posts. Thus, the reader will find it difficult to obtain, evaluate and summarize opinions, and for this there is a need to analyze feelings using the mechanism.

\subsection{Deep learning}

Deep learning is a broad and comprehensive term used for artificial intelligence and machine learning. Deep learning techniques have achieved high levels of success in a diverse and distinct range of areas of application. But the research community lost its interests in the late 1990s in neural networks. Where neural networks are useful in generating text, sentence classification and sentence modeling. Also, deep learning networks have the ability to provide training to supervised and non-supervised groups. Machine learning is part of artificial intelligence that automatically adds benefits from concepts and knowledge to the system for learning without being directly and explicitly programmed. Deep learning algorithms provide opportunities to extract complex data with a high level of abstraction, mainly because they are considered practical only for shallow neural networks "(Neural or two-layer neural networks) as an exercise of a" deep "neural network (neural networks with more layers) is complex and extremely mathematically expensive. Deep learning includes many networks such as CNN (convolutional neural networks), RNN (recurrent neural networks), recursive neural networks, DBN (deep belief networks) and many more.

\section{SENTIMENT ANALYSIS TECHNIQUES}

Sentiment Classification techniques are separated into two different techniques which is ML and Lexicon based Approaches 


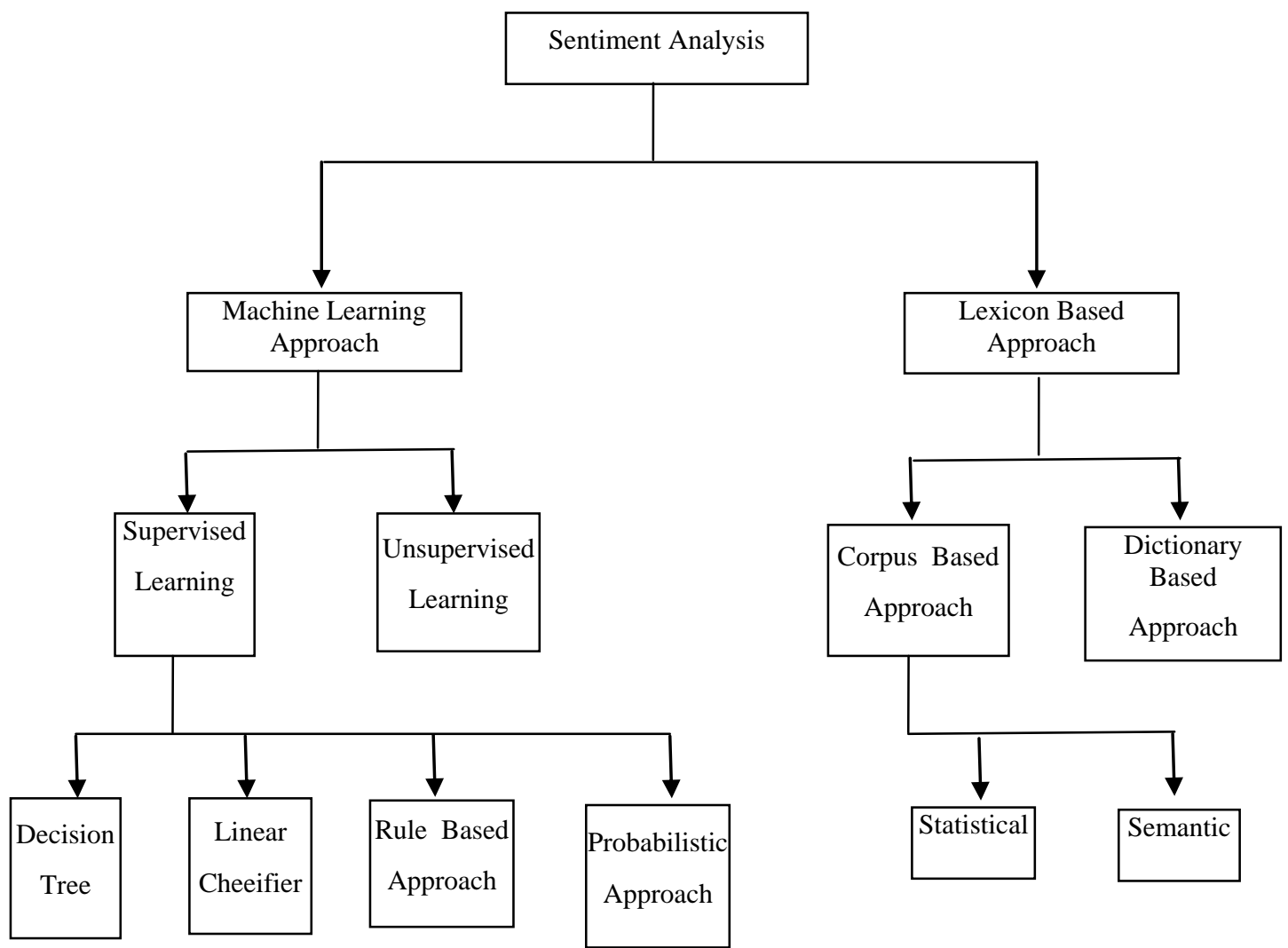

Figure 1 Sentiment Analysis Tech. [5]

Techniques for Sentiment Analysis:

sentiment analysis depends on two types of techniques, which are techniques based on machine learning and on the dictionary.

Techniques based on machine learning: In this type it is applied through extracting sentences and side levels. Features consist of Parts of Speech Marks (POS), n-gram, bi-gram, monogram and bag-of-words. Machine learning also has three aspects of sentence and side: Nave Bayes, Vector (SVM) and Maximum Entropy. and Lexicon-based technologies: In this type, they depend on trees through which decisions are made such as k-Nearest Neighborhood (k-NN), Conditional Random Field (CRF), Hidden Markov Model (HMM), and Class Dimensional Classification (SDC) and Chain Optimization (SMO) related to emotional classification methodologies.

The machine learning approach also has three categories:

a) supervised; b) semi-supervised; and c) non-supervised. This approach is capable of automation and it can handle a large amount of data and therefore it is very suitable for the analysis of sentiment.

In this section, many sentiment analysis studies are discussed using deep learning techniques. This review is also done in the field of emotional analysis based on several recent studies.

\section{SENTIMENT ANALYSIS MODELS}

In order to accurately classify feelings, many researchers have made great efforts to combine the concepts of machine learning and deep learning in recent years. This section describes a summary of the latest to the best of our knowledge researches of the studies related to sentiment analysis of web contents about emotions, user opinions, etc. using deep learning techniques. Morale analysis tasks can be performed effectively by implementing various models such as deep learning models and NLP. These models include CNN (convolutional neural networks), RNN (recursive neural network), DNN (deep neural networks), RNN (repetitive neural networks), and DBN (deep belief networks).

The author [Ahmad Al Sallab, et al, 2015] [13] $\mathrm{He}$ investigated the analysis of sentiment through the comments of Algerian users on various brands pages on Facebook sites of companies in Algeria. They collected 100,000 written comments, and their comments were 25,000 as positive, negative or neutral. $\mathrm{CNN}$ has been implemented as a feature extractor to forecast comment morale. Their model achieves $89.5 \%$ accuracy.

In this study the author [J. Islam, et al, 2016] [1] a new framework is proposed to analyze visual feelings using a transport learning approach to predict confidence. Where excessive parameters of a very deep convolutional neural network were used to form the network model in order to prevent overcoming it, and a lot of investigations were carried out to analyze feelings and elicit feedback from textual contents of social networks where visual content has gained relatively more popularity than textual content among users of different social networks. Also, the ability to classify effective CNN images and transfer that knowledge was used to analyze how the image feels. Therefore, it is suggested that a very deep CNN model consisting of 22 layers be used to learn the visual features of predicting image morale. The learning transfer process and the use of excessive parameters of a very deep network image class have been exploited to create the proposed model to prevent oversupply. The fastest 60-period strategy has been implemented from the network training prototype as GoogleNet has implemented faster than 250 periods. Regarding the experiment, he evaluated the form on 
the Twitter dataset, which is an image dataset based on user tweets containing 1,269 images. A deep CNN model for visual sentiment analysis using Caffe and Python was implemented on Linux X86-64, and the training was conducted by the network using ImageNet dataset to reduce the increase in network data increase and implementation based on image translation and recovery. The network was optimized using the stochastic algorithm. however, AlexNet was used in previous works and GoogleNet made almost 9\% progress on AlexNet performance. By adjusting the Twitter dataset, the proposed model demonstrates a noticeable improvement in performance for visual sentiment analysis.

[Vateekul, et al, 2016] [2] has proposed two deep learning techniques for the sentiment classification of Thai Twitter data, i.e., Convolutional Neural Network (DCNN) and ShortTerm Memory (LSTM). Data processing was conducted properly. Data was collected from the users and their followers of Thai Twitter. After filtering the data, only the users with Thai tweets and tweets with Thai characters were selected. Five experiments were conducted to achieve finest parameters for deep learning, to compare the deep learning with classical techniques and to achieve the words sequence importance. Three-fold cross validation was used to verify the process. The results concluded that the accuracy is high in DNN than LSTM and both techniques of deep learning are higher in accuracy than SVM and Nave Bayes but lesser than Maximum Entropy. Higher accuracies were found in original sentences than shuffled sentences so the words sequence is important.

[Baecchi, et al, 2016] [3] suggested a model that analyzes spirits using the textual and visual contents of social networks. He looked at recent works and saw that models based on neural networks are better than N-gram models, and he also searches for the use of a multimedia method using neural networks to address Analysis of the feelings of microblogging and the motive behind this method is recent developments in training language paradigms based on neural networks for their effectiveness when dealing with text on the web, especially in the grammar and semantic similarity of words; and in uncensored learning. The basis of the scheme is the continuous form of the word bag (CBOW) with negative sampling, which is referred to as CBOW-LR, and it is specialized to classify feelings. This is better than using CBOW directly. It evaluates four data sets, such as Sanders Corpus, Sentiment140, SemEval2013, and SentiBank Twitter. The proposed model outperformed the CBOWS + SVM and FSLM (fully supervised prospect language model). Learning and overcoming big data sets are required to get the best performance.

In this study the author [Ibrahim Rouby. Vateekul, et al, 2016] [4] this research work discussed a sentiment analysis model to analyze the people's opinions and emotions towards some comments collected from the Facebook. The collected comments were written in Arabic as the Arabic language in both challenges and intensity. The work analyzed the impact of preprocessing operations like noise rejection, stemming, and normalization in the user's comments.

In this study the author [Abinash Tripathy, et al, 2016] [9] the reviews and blogs that have been obtained through social networks are important resources for improving decisionmaking and analyzing them. As reviews are inherently disorganized, they need some kind of treatment, such as classification and aggregation, to provide useful information for future uses. Also, machine learning methods help in classifying these reviews. The authors also adopted four different algorithms for classification of feelings and machine learning. These algorithms are: Naïve Bayes, SVM, Maximum Entropy, and Stochastic Gradient Descent. They also looked at some measurable criteria to evaluate the performance of the approved algorithm.

In this study the author [I. Mohan, et al, 2017] [10], one of the tasks for analyzing feelings is to determine the sequence of words affected by negative and is considered an important task. The authors combined the problem of determining the polarity of a sentence with determining the negation range. They also suggested an approach to dealing with negation based on linguistic characteristics that determine the influence of different types of negation. The proposed approach improved the accuracy of determining negation and general morale analysis.

[Zi-Li Zhang, et al, 2017] [7] suggested an LSTM-centered design that was responsive to the words that existed in the vocabulary; therefore, the keywords influence the semantics of the complete document. The suggested model was assessed in a brief-text Sentiment Analysis task on two datasets like IMDB and SemEval2016. Experiential outcomes delineated that the design outperformed the baseline LSTM by $2 \%$ in respect of accuracy and was effectual with notable performance enhancement over numerous non-RNN latent semantic designs (specifically in handling brief texts). It also integrated the idea to an alternative of LSTM named the gated recurrent unit (GRU) model and attained fine performance, which confirmed that this methodology was adequate to augment disparate Deep Learning models.

[Ramy Baly, et al, 2017] [11] the author has explored many network designs based on Deep Auto Encoder Deep Belief and the Recursive Auto Encoder networks. They also do not mention the electrodes classification there. They use the Linguistic Data Consortium Arabic Tree Bank (LDC ATB) dataset and showed that the model far outperforms the latest model in the same dataset approximately $9 \%$ in F degree. You get an accuracy of $74.5 \%$.

[Umar Farooq, et al, 2017] [12] built a deep educational model to discover the polarization of tweets in a classification consisting of 5 levels ranging from very positive to very negative. He also retrieved tweets from 12 Arab countries in 4 different regions (North Africa, the Arab Gulf, Egypt and the Arab East). They collected 470 thousand his twins. The deep learning model also consists of a content layer followed by the LSTM layer. Pre-trained word motifs were applied with Word2Vec's skip-gram template. And $70 \%$ accuracy has been achieved in Egyptian wedding tweets and parties.

[Maha Heikal, et al, 2018] [6] suggested a divide and conquers methodology which initially categorized the sentences into disparate types, then executed the SA separately on sentences as of each type. Especially, it was ascertained that the sentences tend to be utmost intricate if it comprised more sentimental words. Thus, it was suggested to employ an NN centered sequence model to categorize opinionated sentences into 3 types as per the count of targets transpired in a sentence. Each pool of sentences was then supplied to a one-dimension CNN separately for sentimental classification. This approach was appraised on 4 sentimental classification datasets and contrasted with extensive baselines. Experiential outcomes exhibited that: (I) sentence type categorization could augment the performance of sentence level SA; (ii) the suggested approach attains modern outcomes on numerous benchmarking datasets. And Combine CNN and LSTM models to predict the sentiments of Arabic tweets that 
use the Arabic Sentiments Tweets database (ASTD). This model outperforms the most recent F1 deep learning model of $53.6 \%$, achieving $65 \%$ accuracy and a F1 score of $64.46 \%$.

\section{DISCUSSION}

This review includes several studies related to the analysis of sentiment through the use of deep learning models. After analysis, it has been demonstrated that with deep learning methods, feeling can be analyzed in a more effective and accurate way since emotion analysis is used to predict user opinions, deep learning models revolve around predicting or imitating the human mind, deep learning models provide more accuracy than shallow models. Deep learning networks have more hidden layers than normal neural networks that have one or two hidden layers making it better. Private architectural RNNs with gateways such as LSTMs have proven to be extremely powerful in capturing the statistical significance of string inputs. LSTM is the first network to introduce a gateway mechanism, designed to solve the problem of faded gradients and capture long-range dependencies. Deep learning networks are able to provide training in supervised / nonsupervised ways. Deep Learning Networks do not contain human intervention, Morale Analysis also includes many different types of problems. By making minor adjustments to the system, you can install several tasks. Also, this method has some limitations, for example, SVM. It is very expensive for training and requires large sets of data. These complex models can take weeks, unlike LSTM. There are also some models that excel in deep learning models like LSTM. In [6] the author used less data so it's the best one as the author used 10,000 tweets with a high accuracy of $64.46 \%$ compared to [12] the other used 470,000 and with an accuracy of $70 \%$. otherwise in [13] the author collected 100,000 and with an accuracy of $89.5 \%$. Thus, it can save time because feature engineering is not necessary. In contrast to other models that take weeks and are very expensive as in SVM.

Table 1. a summary of the latest to the best of the studies related to sentiment analysis and deep learning.

\begin{tabular}{|c|c|c|c|c|}
\hline $\begin{array}{c}\text { Researcher Name } \\
\text { and Year }\end{array}$ & Model Used & Purpose & Data set & Results \\
\hline P.Vateekul 2016 [2] & $\begin{array}{l}\text { Convolutional } \\
\text { Neural Networks } \\
\text { (DCNN) and short- } \\
\text { term memory } \\
\quad \text { (LSTM) }\end{array}$ & $\begin{array}{l}\text { Sentiment } \\
\text { Analysis on } \\
\text { Thai Twitter } \\
\text { Data }\end{array}$ & $\begin{array}{c}3,813,173 \text { tweets }(33,349 \\
\text { negative tweets and } 140,414 \\
\text { positive tweets). }\end{array}$ & $\begin{array}{c}\text { Higher in accuracy than SVM and Nave Bayes lesser } \\
\text { than Maximum Entropy Higher accuracies in original } \\
\text { sentences than shuffled sentence } 60.8 \%\end{array}$ \\
\hline C.Baecchi 2016 [3] & $\begin{array}{c}\text { Deep Neural } \\
\text { Networks } \\
\text { (CBOW-DA-LR) }\end{array}$ & $\begin{array}{l}\text { Visual and } \\
\text { textual } \\
\text { sentiment } \\
\text { analysis }\end{array}$ & $\begin{array}{l}\text { 4 datasets: sanders corpus, } \\
\text { sentiment } 140 \text {, semEval-2013, } \\
\text { and sentiBank twitter Dataset }\end{array}$ & $\begin{array}{c}\text { CBOW-SA-LR model obtained superior classification } \\
\text { accuracy than previous models. } \\
\text { Accuracy } 79.39 \%\end{array}$ \\
\hline $\begin{array}{l}\text { Ramy Baly } \\
\text { [12] } 2017\end{array}$ & $\begin{array}{l}\text { Sentiment analysis } \\
\text { and Long Short- } \\
\text { term Memory } \\
\text { LSTM }\end{array}$ & $\begin{array}{l}\text { models on } \\
\text { the tweets of } \\
\text { both Egypt } \\
\text { and the UAE }\end{array}$ & $\begin{array}{l}\text { retrieved tweets from } 12 \text { Arab } \\
\text { countries in } 4 \text { different } \\
\text { regions (North Africa, the } \\
\text { Arab Gulf, Egypt and the } \\
\text { Arab East). They collected } \\
470,000 \text { his twins }\end{array}$ & $\begin{array}{c}\text { Results indicate the superior performance of deep } \\
\text { learning models, the importance of morphological } \\
\text { features in Arabic NLP, and that handling dialectal } \\
\text { Arabic leads to diff erent outcomes depending on the } \\
\text { country from which the tweets are collected. Accuracy } 70 \\
\%\end{array}$ \\
\hline $\begin{array}{c}\text { Ibrahim Rouby } 2018 \\
\text { [4] }\end{array}$ & $\begin{array}{c}\text { Sentiment } \\
\text { classification and } \\
\text { Machine Learning }\end{array}$ & $\begin{array}{l}\text { to analyze } \\
\text { the people's } \\
\text { opinions and } \\
\text { emotions } \\
\text { towards }\end{array}$ & $\begin{array}{l}\text { 4-GB memory size and } 2.50 \\
\text { GHZ processing speed. The } \\
\text { model also was run and tested } \\
\text { using three testbeds or } \\
\text { datasets }\end{array}$ & $\begin{array}{l}\text { Sentiment model behaves better using the light stemmer } \\
\text { than using the general one } \\
\text { Accuracy } 70.1 \% \text {, }\end{array}$ \\
\hline Maha Heikal 2019 [6] & $\begin{array}{l}\text { Convolutional } \\
\text { Neural Network } \\
\text { (DCNN) and short- } \\
\text { term memory } \\
\quad(\text { LSTM) }\end{array}$ & $\begin{array}{l}\text { to predict the } \\
\text { sentiments } \\
\text { of Arabic } \\
\text { tweets that } \\
\text { use the } \\
\text { Arabic } \\
\text { Sentiments } \\
\text { Tweets }\end{array}$ & $\begin{array}{c}\text { on smaller datasets } \\
\text { which consists of } 10,000 \\
\text { tweets, distributed among } 4 \\
\text { classes (positive, negative, } \\
\text { neutral and objective). }\end{array}$ & $\begin{array}{c}\text { This model achieves an F1- score of } 64.46 \% \text { which } \\
\text { outperforms the state-of-the-art deep learning models F1- } \\
\text { SCORE of } 53.6 \% \text { on Arabic sentiment tweets dataset } \\
\text { (ASTD). }\end{array}$ \\
\hline
\end{tabular}

\section{CONCLUSION}

sentiment analysis refers to control and prediction of feelings and opinions. In this paper, sentiment analysis is performed using deep learning techniques. As deep learning consists of many effective models, which are used to solve a variety of problems accurately and effectively. Various studies have been discussed in this review to know the successful applications of deep learning in the field of emotional analysis. Many problems in the areas of deep learning and sentiment analysis have been solved by obtaining a high degree of accuracy. Next, we will implement the Kaggle model that was created to analyze Arab sentiments and achieve $96 \%$ accuracy that consists of the CNN layer followed by one LSTM layer and completely connected at the end with the prediction layer with one output class between 3 emotional categories (positive, negative and neutral).

\section{REFERENCES}

[1] J. Islam and Y. Zhang, Visual Sentiment Analysis for Social Images Using Transfer Learning Approach, 2016 IEEE Int. Conf. Big Data Cloud Comput. (BDCloud), Soc. Comput. Netw. (SocialCom), Sustain. Comput. 
Commun., pp. 124130, 2016.

[2] P. Vateekul and T. Koomsubha, A Study of Sentiment Analysis Using Deep Learning Techniques on Thai Twitter Data, 2016.

[3] C. Baecchi, T. Uricchio, M. Bertini, and A. Del Bimbo, A multimodal feature learning approach for sentiment analysis of social network multimedia, Multimed. Tools Appl., vol. 75, no. 5, pp. 25072525, 2016.

[4] Ibrahim Rouby, Performance evaluation of an adopted sentiment analysis model for Arabic comments from the facebook ,2018, pp. 992-1045, 2018.

[5] J. Singh, G. Singh, and R. Singh, A review of sentiment analysis techniques for opinionated web text, CSI Trans. ICT, 2016.

[6] Maha Heikal, Sentiment Analysis of Arabic Tweets using Deep Learning,the 4th International Conference on Arabic Computational Linguistics (ACLing 2018), November 17-19 2018, Dubai, United Arab Emirates

[7] Hu, Fei, Li Li, Zi-Li Zhang, Jing-Yuan Wang, and XiaoFei $\mathrm{Xu}$, "Emphasizing essential words for sentiment classification based on recurrent neural networks", Journal of Computer Science and Technology, vol. 32, no. 4, pp. 785-795, 2017.

[8] Sun, Xiao, Chengcheng Li, and Fuji Ren, "Sentiment analysis for Chinese microblog based on deep neural networks with convolutional extension features", Neurocomputing, vol. 210, pp. 227-236, 2016.

[9] Abinash Tripathy, Ankit Agrawal, Santanu Kumar Rath, "Classification of Sentiment Reviews Using n-gram Machine Learning", Expert Systems with Applications, Vol. 57, PP.11-126, 2016.
[10] I. Mohan, K, Janani, and M. Karthiga, "A Survey on Sentiment Analysis on Social Network Data", The International Journal of Scientific Research in Computer Science, Engineering and Information Technology, Vol.2, Issue 2, PP. 562-568, March-April 2017.

[11] Ramy Baly, Georges El-Khoury, Rawan Moukalled, Rita Aoun, Hazem Hajj, Khaled Bashir Shaban, and Wassim El-Hajj. Comparative evaluation of sentiment analysis methods across arabic dialects. Procedia Computer Science, 117:266-273, 2017.

[12] Umar Farooq, Hasan Mansoor, Antoine Nongaillard, Yacine Ouzrout, Muhammad Abdul Qadir, "Negation Handling in Sentiment Analysis at Sentence Level", The Journal of Computers, Vol. 12, No. 5, PP. 470478, September 2017.

[13] Ahmad Al Sallab, Hazem Hajj, Gilbert Badaro, Ramy Baly, Wassim El Hajj, and Khaled Bashir Shaban. Deep learning models for sentiment analysis in arabic. In Proceedings of the second workshop on Arabic natural language processing, pages 9-17, 2015.

[14] Assia Soumeur, Mheni Mokdadi, Ahmed Guessoum, and Amina Daoud. Sentiment analysis of users on social networks: Overcoming the challenge of the loose usages of the algerian dialect. Procedia computer science, $142: 26-37,2018$

[15] Tao Chen, Ruifeng Xu, Yulan He, and Xuan Wang, "Improving sentiment analysis via sentence type classification using BiLSTM-CRF and CNN", Expert Systems with Applications, 2016.

[16] Liao, Shiyang, Junbo Wang, Ruiyun Yu, Koichi Sato, and Zixue Cheng, "CNN for situations understanding based on sentiment analysis of twitter data", Procedia Computer Science, vol. 111, pp. 376-381, 2017. 